\title{
STELLAR DISTANCES AND HIPPARCOS
}

\author{
A. E. GÓMEZ \\ URA 335 du CNRS, Observatoire de Meudon, DASGAL, \\ F-92195 Meudon, France
}

\begin{abstract}
Hipparcos, the astrometric satellite of the European Space Agency, launched in August 1989, will provide absolute trigonometric parallaxes for about 100000 stars brighter than the limiting V-magnitude 12.5. In spite of the difficulties caused by a faulty orbit, the mission is successfully going on. A lifetime of more than 4 years is expected which will allow to reach the original objectives of the mission: 2 milli-arcsec for stars brighter than V-magnitude 9 and 4 to 5 milli-arcsec for fainter stars. The observing programme is based upon a single, uniquely-defined Input Catalogue. Field and open cluster stars of almost all effective temperatures, chemical compositions and gravities are being observed. Most types of binaries and variable stars are well represented, but Hipparcos is discovering new ones. Significant parallaxes (relatives errors smaller than $20 \%$ ) will be obtained for stars closer than $100 \mathrm{pc}$ (about 36000 stars). Reliable stellar structure models should fit all the possible observational constraints: the main contribution of Hipparcos parallax data will be the huge improvement in the individual luminosities. The determination of stellar masses from the study of visual binaries will also be improved. The preliminary results of the first-year data reduction are an encouraging indication of the expected quality of the final results. On the basis of a mission duration of 4 years, the final results are foreseen for 1995 .
\end{abstract}

\section{OVERVIEW OF THE HIPPARCOS MISSION}

Hipparcos, the astrometric satellite of the European Space Agency (ESA), is devoted to the accurate measurement of the positions, trigonometric parallaxes and proper motions of about 120000 selected stars down to the limiting $V$-magnitude 12.5. A complete description of the pre-launch status mission is given in Perryman et al. (1989a). Launched in August 1989 by Ariane, Hipparcos failed to reach its geostationary orbit because of the failure of its apogee boost motor. A revised mission was implemented and completed by the end of November 1989 (Van der Ha 1992) and the satellite became operational. Hipparcos is orbiting in a highly elliptical orbit (perigee: $500 \mathrm{~km}$, apogee: $36000 \mathrm{~km}$; period: $10 \mathrm{~h} 40 \mathrm{~m}$ ) and valid observations are collected during $65-70 \%$ of the time.

In the nominal mission, the target accuracy on absolute parallaxes varies from 2 milli-arcsec or better for stars brighter than $V$-magnitude of about 9 to 5 milli-arcsec for fainter stars. This pre-launch accuracy prediction was reviewed by Lindegren and Kovalevsky (1992) with regard to the observed performance 
and expected lifetime of the satellite in its present orbit. They concluded that a mission duration of 4 years, until the end of 1993, is necessary in order to reach the nominal expected accuracy. The finite duration of the mission together with the fact that the satellite is continuously and systematically scanning the sky required a preselection of the stars to be observed and a precise a priori knowledge of the stars' position and magnitude. The catalogue containing the programme stars, called the Input Catalogue (Turon et al., 1992a), was compiled within the framework of the european consortium INCA (for details see Perryman and Turon 1989). The stars were selected on the basis of 214 scientific proposals submitted to ESA in 1982 by the international community. This material was supplemented, by the INCA consortium, with a basic list of bright stars referred to as the "survey" necessary for satellite operations and data reductions. The stellar content of the Input Catalogue is given in a section below.

The data obtained by the satellite (photon counts) are processed independently by the two data reduction consortia FAST and NDAC (see Perryman et al., 1989b). The data reductions consist of re-assembling the satellite data into a system of star positions, parallaxes and proper motions. The two catalogues produced by the consortia will be merged into a single one to be released to the astronomical community.

A byproduct of the astrometric mission is the determination of the Hipparcos $H_{P}$-magnitude for each star with high accuracy. The $H_{P}$-magnitude is the magnitude corresponding to the large bandwith of the Hipparcos detector (Mignard et al., 1989). It is defined by $H_{P}=V+0.408(B-V)-0.130(B-V)^{2}$, the expected accuracies vary from $0^{m} .001$ up to $H_{P}$-magnitude 6 to $0^{m} .01$ at $H_{P}$-magnitude 11 . Moreover, in the process of signal analysis, information on the duplicity or multiplicity of the observed stars is also obtained.

In spite of the difficulties encountered at the beginning, the mission is now successfully going on. On the basis of a mission duration of 4 years, the final Catalogue is foreseen for 1995.

\section{TYPES OF STARS OBSERVED BY HIPPARCOS}

The Input Catalogue, which contains the 118209 programme stars, constitutes the best compromise between the scientific goals of the proposals and the satellite's technical constraints (Turon et al., 1992b). Hipparcos is observing a large variety of stars: field stars of different masses, effective temperatures, chemical compositions and gravities, most types of binary stars and stars in open clusters. Table I summarizes the principal types of stars observed by Hipparcos. As can be seen from this table, different groups of spectroscopically peculiar stars and of variable stars are represented in the observing programme.

Among the observed stars, it is to be noticed that Hipparcos is measuring all the stars brighter than $V$-magnitude 7 , a survey of about 52000 stars and nearby stars selected from specific catalogues such as the Gliese Catalogue (Gliese 1969, Gliese and Jahreiß 1979) and the LHS and NLTT Catalogues (Luyten 1976, 1979, 1980). As stated before, the "survey" is a basic list of bright stars distributed over the whole sky, complete to a given limiting magnitude which is a function 
TABLE I Types of stars observed by Hipparcos.

$\begin{array}{lll}\text { A-type stars } & \text { Eruptive variable stars } & \text { R stars } \\ \text { A stars (H-line emission) } & \text { F-type stars } & \text { R Cr B stars } \\ \text { Am stars } & \text { G-type stars } & \text { RR Lyrae stars } \\ \text { Ap stars } & \text { H- and K- emission stars } & \text { RS CVn stars } \\ \text { B-type stars } & \text { He abnormal stars } & \text { S stars } \\ \text { Barium stars } & \text { Herbig Ae and Be stars } & \text { Semi-regular var. stars } \\ \text { Be stars } & \text { Horizontal branch stars } & \text { Symbiotic stars } \\ \beta \text { CMa stars } & \text { Irregular variable stars } & \text { Sub-dwarf stars (cool) } \\ \text { Carbon stars } & \text { K-type stars } & \text { Sub-dwarf stars (hot) } \\ \text { Cataclysmic variable stars } & \lambda \text { Bootis stars } & \text { T Tauri stars } \\ \text { Cepheid stars } & \text { Li rich stars } & \text { VV Cephei stars } \\ \text { CH like star } & \text { Long-period variable stars } & \text { Weak G-band stars } \\ \text { CH strong stars } & \text { M-type stars } & \text { Weak metal stars } \\ \text { CJ stars } & \text { Marginal barium stars } & \text { White dwarfs } \\ \text { CS stars } & \text { Mira Ceti stars } & \text { Wolf-Rayet stars } \\ \delta \text { Delphini stars } & \text { O-type stars } & \text { W UMa stars } \\ \delta \text { Scuti stars } & \text { Planetary nebulae } & \text { WW Virginis stars } \\ \text { Early-type H-poor stars } & \text { Pulsating variable stars } & \text { X-ray binaries } \\ & & \end{array}$

of the galactic latitude "b" and the spectral type (Crifo et al., 1985):

$\mid \begin{array}{ll}V \leq 7.9+1.1 \sin |b| & \text { for spectral types earlier than or equal to G5 } \\ V \leq 7.3+1.1 \sin |b| & \text { for spectral types later than G5. }\end{array}$

The survey was required for satellite operations and data reductions but it constitutes a sample highly suitable for future statistical studies.

Binary stars of different spectral types are being observed: about 1000 visual binaries, 1000 spectroscopic binaries and 100 eclipsing binaries (Gómez et al., 1989). These figures are underestimated because they concern only the stars specifically proposed as binaries. The Input Catalogue includes about 16000 systems of double or multiple stars, only a part of them being physical systems. Hipparcos is discovering and measuring a large number of non-single stars. After the processing of one year Hipparcos data, the FAST consortium has detected about 7000 new double stars (Mignard et al., 1992). The data reduction task is able to recognize double stars down to a separation of 0 ".12 with $\Delta \mathrm{m}$ up to 3 mag. Most of these new detected doubles have separations smaller than about 17.5 , corresponding probably to physical pairs. Moreover, according to Dommanget (1985), closer systems having separations between 0 ".01 and 0 ". 1 will also be recognized by oscillations of their photocentre during the lifetime of the satellite (astrometric pairs).

Finally, about 1500 stars belonging to 250 open clusters are being observed. The number of observed stars per cluster varies from 1 star to more than 200 (Mermilliod and Turon 1989).

Almost all the observed stars are closer than $1 \mathrm{kpc}$ from the sun. An estimation of the expected heliocentric distance distribution for different categories 
of spectral type is given in Table II. About 3000 stars are missing in the Table because they have no known spectral type and, consequently, no distance estimation. The distances (in pc) were estimated from the spectral type (and the luminosity class if available) and the apparent $V$-magnitude. A reddening correction was applied using the tridimensional model of the galactic interstellar extinction built by Arenou et al. (1992). Hipparcos will provide individual distances for more than 100000 stars closer than about $500 \mathrm{pc}$, distance at which the distance relative error will be of $100 \%$.

TABLE II Number of observed stars, as a function of heliocentric distance (in pc) and spectral type.

\begin{tabular}{|c|rrrr|r|}
\hline Spectral Type & $\leq 100$ & $100-500$ & $500-1000$ & $>1000$ & Total \\
\hline O-B & 140 & 6050 & 2660 & 1760 & 10610 \\
A0-A9 & 1800 & 15240 & 580 & 180 & 17800 \\
F0-F9 & 13000 & 12070 & 110 & 170 & 25350 \\
G0-G9 & 15610 & 6180 & 360 & 210 & 22360 \\
K0-M0 & 4860 & 24100 & 3760 & 480 & 33200 \\
M1-M8 & 490 & 1260 & 680 & 270 & 2700 \\
Other & 670 & 1550 & 480 & 500 & 3200 \\
& & & & & \\
\hline
\end{tabular}

\section{ACCURACY PREDICTION ON PARALLAXES}

Detailed accuracy prediction was carried out before launch based on extensive computer simulations of the instrument, the observations and the data reductions (Lindegren 1989). The pre-launch accuracy prediction has been revised by Lindegren and Kovalevsky (1992) taking into account the observed performance and expected lifetime of Hipparcos in its present orbit. Table III shows the predicted standard errors (in milli-arcsec) on parallaxes as a function of the $H_{P}$-magnitude for a mission duration of 4 years. Following Lindegren and Kovalevsky (1992), the quoted expected external errors have been evaluated by increasing the formal errors by $30 \%$ which represents an upper limit to the degree of uncertainty in the accuracy prediction. Recently, the external errors on the parallaxes obtained by the Data reduction consortia after the processing of one year Hipparcos data have been estimated by various methods: comparison with spectroscopic and photometric parallaxes or using cluster stars and Magellanic Clouds stars (Arenou 1992). These preliminary results show that the external errors are close to the predicted formal errors which means that the figures quoted in Table III are probably overestimated. The quoted values are sky averages: they also depend on the ecliptic latitude. The factor to be applied to the values given in Table III varies from 1.17 on the ecliptic to 0.71 at the poles. Taking into account all these considerations, the expected accuracies on 
the Hipparcos parallaxes will vary between 1 milli-arcsec and 5 milli-arcsec.

TABLE III Predicted standard errors (in milli-arcsec) of parallaxes as a function of $H_{P}$-magnitude for a mission duration of 4 years.

\begin{tabular}{|c|ccccccc|}
\hline$H_{P}$ & $\leq 6.5$ & $6.5-7.5$ & $7.5-8.5$ & $8.5-9.5$ & $9.5-10.5$ & $10.5-11.5$ & $>11.5$ \\
\hline$\sigma_{\pi}$ & 1.2 & 1.3 & 1.6 & 2.0 & 2.8 & 3.7 & 4.6 \\
\hline
\end{tabular}

Let us compare the parallaxes expected from the Hipparcos mission with those presently available. The most complete source of ground-based trigonometric parallaxes is the New Yale Catalogue from van Altena et al. (1992). It contains 7881 stars with accuracies ranging from 0.5 milli-arcsec to 25 milliarcsec or higher: a typical value is 15 milli-arcsec. Hipparcos is not only measuring a larger number of parallaxes (about 100000), but the main point is that the Hipparcos parallaxes will be absolute.

\section{HIPPARCOS CONTRIBUTION TO THE STELLAR STUCTURE}

Among the observable quantities used in modeling stellar interiors, luminosity and mass (determined from the study of visual binaries) depend on distance determination.

The main contribution of the parallaxes measured by Hipparcos will be the significant improvement on stellar luminosities. Adopting the expected average standard error on the measured parallax ( 2 milli-arcsec), stars closer than $100 \mathrm{pc}$ will have parallax relative errors $\left(\frac{\sigma_{\pi}}{\pi}\right)$ smaller than or equal to $20 \%$. The corresponding expected errors on absolute magnitudes $\left(\varepsilon_{M}=2.17 \frac{\sigma_{\pi}}{\pi}\right)$ due to the error in the parallax as a function of the distance are given in Table IV.

TABLE IV Expected errors on absolute magnitudes.

\begin{tabular}{|r|c|l|}
\hline $\mathrm{r}(\mathrm{pc})$ & \multicolumn{1}{|c|}{$\frac{\sigma_{\pi}}{\pi}$} & \multicolumn{1}{|c|}{$\varepsilon_{M}$} \\
\hline 5 & $1 \%$ & $0^{m} .02$ \\
10 & $2 \%$ & $0^{m} .04$ \\
20 & $4 \%$ & $0^{m} .09$ \\
50 & $10 \%$ & $0^{m} .2$ \\
100 & $20 \%$ & $0^{m} .4$ \\
\hline
\end{tabular}


Let us compare these figures with those presently available using a few examples:

- Nearby stars closer than 20 pc. Nearby stars populate the H-R diagramme essentially on the main sequence, corresponding to stars having masses between 0.3 and $2 m_{\odot}$. The sample also contains a few stars of different types: slightly evolved stars, subgiants, giants and white dwarfs. Hipparcos is observing about 1100 stars contained in the Gliese Catalogue of nearby stars (Gliese 1969, Gliese and Jahreiss 1979). Adopting for these stars the errors on the visual absolute magnitudes quoted in the Third version of the Gliese and Jahreiß Catalogue of nearby stars (Gliese and Jahreiss 1992), only a few of them have errors smaller than $0^{m} .1$. For the same stars, all the absolute magnitudes calculated with the Hipparcos parallaxes will have errors smaller than $0^{m} .1$. In particular, powerful tests for the input physics of the stellar interior models will become possible (Baglin 1988). Recently, Lebreton et al. (1992) have modeled a close binary system and found some inconsistencies between the adopted physical model and the observational data.

- H-R diagrammes of open clusters. H-R diagrammes of cluster stars provide well-known tests for the stellar structure theory. Hipparcos will provide a direct distance determination for about 15 galactic clusters. Table $V$ gives the expected individual and mean parallax errors for the observed clusters closer than $250 \mathrm{pc}$. The distances were taken from the Cluster Data Base of Mermilliod (1988). The clusters are ordered by increasing distances. The individual and mean parallax relative errors are given in the fourth and fifth columns, respectively. The indicated precisions take into account the dependence on ecliptic latitude. The figures quoted for the mean parallax precision were calculated using the fact that for stars observed within a small area on the sky, like stars in clusters, a correlation of parallax results exists. In this case the expected improvement by averaging over $N$ stars is given roughly by $\sigma_{\langle\pi\rangle}=\sigma_{\pi} \times N^{-0.35}$ (Lindegren 1989). Most of these clusters will have mean parallax relative errors smaller than $20 \%$.

- Halo stars. Hipparcos does not observe globular cluster stars but it is observing different types of Population II stars, in particular about 700 sub-dwarf stars of various metallicities. At present, less than 30 sub-dwarf stars have parallax relative errors smaller than or equal to $20 \%$. This number will be multiplied by 10 at the end of the Hipparcos mission.

On the other hand, from the 100000 parallaxes measured by Hipparcos, mean absolute magnitudes with precisions ranging between $0^{m} .01$ to $0^{m} .2$ (Mennessier 1988) will be obtained for a large number of stellar types.

Hipparcos parallaxes will contribute to improve the determination of stellar masses from the study of visual binaries. For pairs with known orbits, the sum of masses of the components will be improved. However, for stellar structure modeling, individual masses are required. About 80 systems closer than $20 \mathrm{pc}$ are observed by the satellite, but according to Popper (1980) only half of them have definitive or reliable orbits and known mass ratios. The spectral type distribution of the bright companions for these pairs shows that the concerned stars 
TABLE V Cluster stars: individual and mean parallax precisions.

\begin{tabular}{|c|rrrrr|}
\hline Name & $\mathrm{r}(\mathrm{pc})$ & $N^{a}$ & $\frac{\sigma_{\pi}}{\pi}$ & $\frac{\sigma_{\langle\pi\rangle}}{\langle\pi\rangle}$ & S.T. $^{b}$ \\
& & & & & \\
\hline Hyades & 48 & 209 & $11 \%$ & $2 \%$ & A2 \\
Coma Ber & 79 & 117 & $17 \%$ & $3 \%$ & A0 \\
Pleiades & 125 & 74 & $29 \%$ & $6 \%$ & B5 \\
IC 2602 & 151 & 34 & $23 \%$ & $7 \%$ & B0 \\
IC 2391 & 153 & 21 & $21 \%$ & $7 \%$ & B3 \\
Praesepe & 158 & 22 & $37 \%$ & $12 \%$ & A0 \\
$\alpha$ Per & 164 & 133 & $36 \%$ & $6 \%$ & B2 \\
NGC 6475 & 239 & 12 & $56 \%$ & $23 \%$ & B8 \\
Blanco 1 & 240 & 18 & $52 \%$ & $19 \%$ & B5 \\
& & & & & \\
\hline
\end{tabular}

${ }^{a} N$ is the number of observed stars

${ }^{b}$ Spectral type of the hottest star on the main sequence

have masses around the solar mass. The number of stars for each spectral type is given between parenthesis: $A(2), F(8), G(12), K(6)$ and $M(8)$. Moreover, only a few of these pairs will get individual masses with a relative accuracy smaller than 5\%. According to Morel (1988), the Hipparcos positions available for each component during the mission will allow to derive the masses of the components for a larger number of systems. The simulations carried out by him showed that for about 110 systems individual masses will be derived with a relative accuracy better than $15 \%$ and for 25 systems better than $5 \%$. Recently, Dommanget and Lampens (1992) discussing the expected contributions of Hipparcos to the study of double stars found less optimistic results.

\section{CONCLUDING REMARKS}

From a technical point of view, Hipparcos may continue to collect high quality measurements until roughly June 1994. This well-defined limit to the satellite lifetime is larger than the mission duration necessary to get the target accuracy on absolute parallaxes. First preliminary results obtained by both Data reduction consortia, after processing one year data, are an encouraging indication of the expected quality of the final results. Lindegren (1992) built an H-R diagramme using about 3000 stars with relative (formal) parallax errors smaller than $10 \%$. Nevertheless, any star in the diagramme may have a larger parallax error than given by the formal errors, since one year of observation is rather too short to separate the astrometric parameters (parallax and proper motion).

Improvements are expected for luminosities of individual and cluster stars and for luminosities and masses of stars in visual binaries. They should allow 
better qualitative and quantitative tests on stellar interior models.

\section{ACKNOWLEDGMENTS}

I would like to thank F. Arenou, D. Morin and D. Stefanovitch for their technical assistance in the preparation of this paper.

\section{REFERENCES}

Arenou, F., Grenon, M., and Gómez, A. 1992, $A \mathscr{G} A$, 258, 104

Arenou, F. 1992, Private communication

Baglin, A. 1988, in Scientific Aspects of the Input Catalogue Preparation, II, Sitges, Jan. 1988, J. Torra \& C. Turon (eds), 103

Crifo, F., Turon, C., and Grenon, M. 1985, ESA SP-234, 67

Dommanget, J. 1985, Ap\&SS, 110, 47

Dommanget, J., and Lampens, P. 1992, ApESS, submitted

Gliese, W. 1969, Veroff. Astron. Rechen-Instituts, Heidelberg, No 22

Gliese, W., and Jahreiß, H. 1979, $A \& A S, \mathbf{3 8}, 423$

Gliese, W., and Jahreiß, H. 1992, Third Catalogue of Nearby Stars, CD-ROM version

Gómez, A., Crifo, F., and Turon, C. 1989, in ESA SP-1111, vol. II, 89

Lebreton, Y., Auvergne, M., Morel, P., and Baglin, A. 1992, in Inside the Stars, I.A.U. Colloquium No 137, Vienna (Austria), April 1992

Lindegren, L. 1989, in ESA SP-1111, vol. III, 311

Lindegren, L. 1992, ESA SP-349, (in press)

Lindegren, L., and Kovalevsky, J. 1992, Highlights in Astronomy, vol. 9 (in press)

Luyten, W.J. 1976, LHS Catalogue, Minnesota (and revised version)

Luyten, W.J. 1979, NLTT Catalogue, Minnesota

Luyten, W.J. 1980, NLTT Catalogue, Minnesota

Mennessier, M.O. 1988, in Scientific Aspects of the Input Catalogue Preparation, II, Sitges, Jan. 1988, J. Torra \& C. Turon (eds), 113

Mermilliod, J.C. 1988, Bull. Inf. CDS, 31, 175

Mermilliod, J.C., and Turon, C. 1989, in ESA SP-1111, vol. II, 177

Mignard, F., Foeschlé, M., Falin, J.L., Andreasen, G. K., Høg, E., Grewing, M., and Scales, D. 1989, in ESA SP-1111, vol. III, 205

Mignard, F., Badiale, M., Bernacca, P.L., Bernstein, H., Falin, J.L., Froeschlé, M., Hering, R., and Prezioso, G. 1992, in Complementary Approaches to Double and Multiple Star Research, I.A.U. Colloquium No 135, Pine Mountain (USA), April 1992 
Morel, P. 1988, in Scientific Aspects of the Input Catalogue Preparation, II, Sitges, Jan. 1988, J. Torra \& C. Turon (eds), 235

Perryman, M.A.C., and Hassan, H. (eds) 1989a, ESA SP-1111, vol. I

Perryman, M.A.C., Lindegren, L., Murray, C.A., Høg, E., and Kovalevsky, J.

(eds) 1989b, ESA SP-1111, vol. III

Perryman, M.A.C., and Turon C. (eds) 1989, ESA SP-1111, vol. II

Popper, D.M. 1980, ARAEBA, 18, 115

Turon, C., Crézé, M., Egret, D., Gómez, A., Grenon, M., Jahreiß, H., Réquième, Y., Argue, A.N., Bec-Borsenberger, A., Dommanget, J., Mennessier, M.O. et al. 1992a, ESA SP-1136, 7 vol.

Turon, C., Gómez, A., Crifo, F., Crézé, M., Perryman, M.A.C., Morin, D., Arenou, F., Nicolet, B., Chareton, M., and Egret, D. 1992b, $A \& A$, 258, 74

van Altena, W.F., Lee, J.T., and Hoffleit, E.D. 1992, The General Catalogue of Trigonometric Stellar Parallaxes, Yale University Observatory, CD-ROM preliminary version

van der Ha, J. 1992, ESA bulletin, 69, 9 